\title{
ON THE PERFORMANCE OF SUBSPACE METHODS WITH ARRAY MODEL ERRORS AND SPATIAL SMOOTHING
}

\author{
K. V. S. Hari \\ Dept. of ECE, Indian Institute of Science \\ Bangalore - 560 012, India \\ e-mail: hari@ece.iisc.ernet.in
}

\author{
Uma Gummadavelli \\ Dept. of EE, Auburn University, \\ Auburn, $A L-\$ 6859$, USA \\ guma@eng.auburn.edu
}

\begin{abstract}
In this paper, the effect of spatial smoothing (Forward smoothing and Forward-Backward smoothing) on the performance of subspace methods in the presence of Array Model errors for Direction-Of-Arrival (DOA) estimation is studied. Theoretical expressions for the Mean Squared Error (MSE) in DOA are obtained, based on a common framework of analysis. Simulations are carried out to substantiate the theory developed. For the cases considered, smoothing improves the performance of ESPRIT and Minimum-Norm method while it is not so for MUSIC.
\end{abstract}

\section{INTRODUCTION}

Estimation of the Direction Of Arrival (DOA) of highly correlated/coherent sources impinging upon a Uniform Linear Array(ULA) of sensors by the subspace methods (MUSIC,Min-Norm method,ESPRIT), is done by using a spatially smoothed covariance matrix (Forward Smoothed(FS) and Forward-Backward smoothed(FBS)). In practice, the sensor characteristics (gain and phase responses, positions etc.) are not ideal leading to $\boldsymbol{A r}$ ray Model Errors. These errors contribute to a perturbation in the covariance matrix resulting in a perturbation of the subspaces and therefore an error in the estimated DOA. The performance study in the case of a Forward covariance matrix $\left(\mathbf{R}_{f}\right)$ (no spatial smoothing) due to Array Model Errors has been carried out in $[1,2,3,4,5]$. In this paper, we study the effect of spatial smoothing on the asymptotic performance of the subspace methods in the presence of Array Model Errors. The performance measure chosen is the Mean Squared Error (MSE) in the DOA. Theoretical expressions for the MSE in DOA are obtained for each of the subspace methods, which can be used to analyze any type of array model error given the statistics of the model error substantiated by numerical experiments.

\section{BACKGROUND}

Consider the scenario of $M$ possibly coherent narrowband plane waves (with a wavelength $\lambda_{0}$ ) incident on a ULA of $L_{1}$ sensors (with an interelement spacing of $d$ ) from various directions $\theta_{i}$. The subspace methods determine the DOA from the covariance matrix of the array output vector which can be represented as ${ }^{1}$ $\mathbf{R}_{f}=\mathbf{A S A} \mathbf{A}^{H}+\sigma_{n}^{2} \mathbf{I}$ with $\left.\mathbf{A}=\sqrt{L_{1}}\left[\mathbf{a}_{1}, \mathbf{a}_{2} \cdots, \mathbf{a}_{M}\right)\right]$, where $\mathbf{a}_{i}=\left(1 / \sqrt{L}_{1}\right)\left[1, e^{j \omega_{i}}, \cdots, e^{j\left(L_{1}-1\right) \omega_{i}}\right]^{T}$, is the normalised array steering vector, $\omega_{i}=2 \pi\left(d / \lambda_{0}\right) \sin \theta_{i}$, $\mathbf{S}$ is the source covariance matrix, $\sigma_{n}^{2}$ is the variance of the additive noise at the output of the sensor array and $I$ the identity matrix. In the FS approach, the array is divided into $K$ subarrays of $L$ sensors each $\left(K=L_{1}-L+1\right)$ and $\mathbf{R}_{f s}=\frac{1}{K} \sum_{p=1}^{K} \mathbf{W}_{p} \mathbf{R}_{f} \mathbf{W}_{p}^{H}=$ $A_{L} S_{f} A_{L}^{H}+\sigma_{n}^{2} I$ where $W_{p}=\left[0_{(p-1) \times L}\left|I_{L}\right| 0_{(K-p) \times L}\right]$, $A_{L}$ denotes the subarray array steering matrix and $\mathbf{S}_{f}$ the smoothed source covariance matrix $[6,7]$. Given a perturbed covariance matrix the performance of the subspace methods has been characterized using a common framework of analysis which relates the MSE in DOA directly to the statistics of the perturbed covariance matrix $[6,7]$. The expressions for MSE depend upon the terms ${ }^{2} \Gamma_{\alpha_{i} \alpha_{i} \beta_{i} \beta_{i}}, \Gamma_{\alpha_{i} \beta_{i} \alpha_{i} \beta_{i}}$ where $\beta_{i}=$ $\mathbf{R}_{i}^{+} \mathbf{a}\left(\omega_{i}\right), \mathbf{R}_{s}=\mathbf{A}_{L} \mathbf{S}_{f}, \mathbf{A}_{L}^{H}$ and $\alpha_{i}$ is a noise subspace vector corresponding to the subspace method $\left(=\mu_{i}\right.$ for MUSIC, $=\nu_{i}$ for Min-Norm method, $=\epsilon_{i}$ for ESPRIT) $[6,7]$.

For example, the MSE expressions for MUSIC are given as $[6,7]$ with $\mu_{i}=P_{n} d_{i}, P_{n}=I-A A^{+}$,

\footnotetext{
"In this paper, the overbar "-" will be used to denote the expectation operator. The superscripts used are $\mathrm{T}$ for transpose, + to for the pseudoinverse, * for complex conjugate and $H$ for complex conjugate transpose. $j$ denotes $\sqrt{-1}$ and $\delta_{k l}=1$, for $\boldsymbol{k}=\boldsymbol{l}$ and zero otherwise. $\hat{x}$ denote an estimate of $x, \Delta x$ indicates an error in the parameter $x, \operatorname{Cov}(X, Y)=\overline{(X-\bar{X})(Y-\bar{Y})^{H}}$

${ }^{2} \Gamma_{\text {uvw }}=\operatorname{Cov}\left(\mathbf{u}^{H} \Delta \mathrm{Rw}, \mathbf{v}^{H} \Delta \mathbf{R x}\right), \mathbf{u}, \mathrm{v}, \mathrm{w}, \mathrm{X}$ are vectors of appropriate dimenaion.
} 


$$
\begin{aligned}
& \mathrm{d}_{\mathbf{i}}=\left.(\partial \mathbf{a}(\omega) / \partial \omega)\right|_{\omega=\omega_{i}}, \\
& \overline{\left(\Delta \omega_{i}\right)_{m u}^{2}}=\operatorname{Re}\left(\Gamma_{\mu_{i} \mu_{i} \beta_{i} \beta_{i}}+\Gamma_{\mu_{i} \beta_{i} \mu_{i} \beta_{i}}\right) /\left(2\left(\mu_{i}^{H} \mu_{i}\right)^{2}\right),
\end{aligned}
$$

The above expressions are general and are applicable to any type of perturbation in the covariance matrix.

\section{PERFORMANCE ANALYSIS}

The perturbed asymptotic covariance matrix considered in this paper is given by [2]

$$
\hat{\mathbf{R}}=(\mathbf{A}+\Delta \mathbf{A}) \mathbf{S}(\mathbf{A}+\Delta \mathbf{A})^{H}+\sigma_{n}^{2} \mathbf{I}
$$

The effect of finite data is not considered in this paper. The steps involved in the analysis can be outlined as follows

1. Perturb the array model and obtain the expression for $\Delta \mathbf{R}$ and subsequently for $\alpha_{i}^{H} \Delta \mathbf{R} \beta_{i}$, for the type of $\mathbf{R}$ (FS,FBS) chosen for analysis.

2. Obtain the general expressions for $\Gamma_{\alpha_{i} \alpha_{i} \beta_{i} \beta_{i}}$, $\Gamma_{\alpha_{i} \beta_{i} \alpha_{i} \beta_{i}}$ in terms of the statistics of $\Delta \mathbf{A}_{\text {; }}$ to study the performance of a method, use the appropriate $\alpha_{i}$ in the MSE expressions.

The general MSE expressions for each of the smoothed covariance matrices are given below.

Theorem 1 [8] For the FS case, $\left(\Gamma_{\alpha_{i} \alpha_{i} \beta_{i} \beta_{i}}\right)_{f s}=$ $\alpha_{i}^{H} B_{1 f s} \alpha_{i},\left(\Gamma_{\alpha_{i} \beta_{i} \alpha_{i} \beta_{i}}\right)_{f s}=\alpha_{i}^{H} \mathbf{B}_{2 f,} \alpha_{i}^{*}$, with

$\mathbf{B}_{1 f q}=\frac{1}{K^{2}} \sum_{p=1}^{K} \sum_{q=1}^{K} \mathbf{W}_{p} \sum_{r=1}^{M} \sum_{t=1}^{M} \beta_{i p}(r) \beta_{i q}^{*}(t) \overline{\Delta \mathbf{a}_{r} \Delta \mathbf{a}_{t}^{H}} \mathbf{W}_{q}^{H}$,

$\mathbf{B}_{2 f s}=\frac{1}{K^{2}} \sum_{p=1}^{K} \sum_{q=1}^{K} \mathbf{W}_{p} \sum_{r=1}^{M} \sum_{t=1}^{M} \beta_{i p}(r) \beta_{i q}(t) \overline{\Delta \mathbf{a}_{r} \Delta \mathbf{a}_{t}^{T}} \mathbf{W}_{q}^{T}$,

with $\beta_{i p}=\left(\beta_{i p}\right)_{f s}=\frac{1}{\sqrt{L}} \mathbf{S} \psi^{(p-1)} \mathbf{S}_{f s}^{-1} \mathbf{i}_{i}$,

$\psi=\operatorname{diag}\left(\exp \left(j \omega_{i}\right)\right), i=1,2, \cdots, M$ and $\alpha_{i}$ representing $\mu_{i}, \nu_{i}, \epsilon_{i}$.

Proof : The perturbed $\mathbf{R}_{f s}$ is given by

$\hat{\mathbf{R}}_{f s}=\frac{1}{K} \sum_{p=1}^{K} \mathbf{W}_{p}\left((\mathbf{A}+\Delta \mathbf{A}) \mathbf{S}(\mathbf{A}+\Delta \mathbf{A})^{H}+\sigma_{n}^{2} \mathbf{I}\right) \mathbf{W}_{p}^{H}$

where $\triangle \mathbf{A}$ corresponds to the full array. Neglecting second order terms, $\Delta \mathbf{R}_{f \triangleleft} \approx \frac{1}{K} \sum_{p=1}^{K}\left(\mathbf{W}_{p} \Delta \mathbf{A S} \psi^{(p-1)} \mathbf{A}_{L}^{H}+\right.$ $\left.\mathbf{A}_{L} \psi^{-(p-1)} \mathbf{S} \Delta \mathbf{A}^{H} \mathbf{W}_{p}^{H}\right)$. Then

$$
\begin{aligned}
\alpha_{i}^{H} \Delta \mathbf{R}_{f o} \beta_{i} & =\frac{1}{\sqrt{L} K} \sum_{p=1}^{K} \alpha_{i}^{H} \mathbf{W}_{p} \Delta \mathbf{A S} \psi^{(p-1)} \mathbf{S}_{f,}^{-1} \mathbf{i}_{i} \\
& =\alpha_{i}^{H} \frac{1}{K} \sum_{p=1}^{K} \mathbf{W}_{p} \sum_{r=1}^{M} \beta_{i p}(r) \Delta \mathbf{a}_{r}
\end{aligned}
$$

$$
\overline{\Delta \mathbf{a}_{r}\left(\omega_{i}\right) \Delta \mathbf{a}_{t}^{H}\left(\omega_{j}\right)}=\sigma_{r}^{2} I \delta_{i j} \delta_{r t}, \overline{\Delta \mathbf{a}_{r}\left(\omega_{i}\right) \Delta \mathbf{a}_{t}^{T}\left(\omega_{j}\right)}=0 .
$$

Gain and Phase Errors: The gain and phase responses of the sensors are assumed to deviate from the nominal response and can be expressed as $[2,3]$

$$
\Delta \mathbf{A}=\left[\Delta \mathbf{G}_{1} \mathbf{a}\left(\omega_{1}\right)\left|\Delta \mathbf{G}_{2} \mathbf{a}\left(\omega_{2}\right)\right| \cdots \mid \Delta \mathbf{G}_{M} \mathbf{a}\left(\omega_{1}\right)\right]
$$

where $\Delta \mathbf{G}_{i}$ is assumed to be a diagonal matrix. If the effect of mutual coupling is considered, $\Delta G_{i}$ has off-diagonal elements too. Consider the perturbations in the gain and phase response to be small enough, independent of the DOA, and modelled as zero-mean, uncorrelated random variables with variances $\sigma_{a}^{2}, \sigma_{p}^{2}$ respectively $[2,3]$, then the statistics of the Angle Independent Errors are given as

$\overline{\Delta a_{r} \Delta a_{t}^{H}}=\sigma_{g}^{2}\left(I \bigodot a_{r} a_{t}^{H}\right), \overline{\Delta a_{r} \Delta a_{t}^{T}}=\sigma_{d}^{2}\left(I \bigodot a_{r} a_{t}^{T}\right)$ 
where $\odot$ denotes the Hadamard (or Schur) product of matrices and $\sigma_{g}^{2}=\sigma_{a}^{2}+\sigma_{p}^{2}, \sigma_{d}^{2}=\sigma_{a}^{2}-\sigma_{p}^{2}$. In the case of Angle dependent errors (as in the case of sensor position errors), the variances are assumed to be DOA dependent.

Using the statistics of the errors given above and the expressions given in Theorems 1 and 2, the performance of the methods is studied in this paper. Due to lack of space, the simplified expressions are given only for MUSIC when Gain and Phase errors are considered. $\overline{\left(\Delta \omega_{i}\right)_{m u}^{2}}=\frac{1}{2 K^{2} L|| \mu_{i} \|^{4}}\left(\sigma_{g}^{2} \mu_{i}^{H} \mathbf{B}_{g p} \mu_{i}+\sigma_{p}^{2} R e \mu_{i}^{H} \mathbf{C}_{g p} \mu_{i}^{*}\right)$, where

$$
\begin{aligned}
& \mathbf{B}_{g p}=\sum_{p=1}^{K} \sum_{q=1}^{K} \mathrm{~W}_{p}\left(\mathrm{I} \bigodot\left(A \boldsymbol{\beta}_{i p}\right)\left(A \beta_{i q}\right)^{H}\right) \mathbf{W}_{q}^{H} \\
& \mathbf{C}_{g p}=\sum_{p=1}^{K} \sum_{q=1}^{K} \mathbf{W}_{p}\left(\mathrm{I} \bigodot\left(A \boldsymbol{\beta}_{i p}\right)\left(A \beta_{i q}\right)^{T}\right) \mathbf{W}_{q}^{T}
\end{aligned}
$$

Similar expressions can be easily obtained for ESPRIT and Minimum-Norm methods [8].

\section{RESULTS AND CONCLUSIONS}

MSE in DOA obtained through simulations (averaged over 200 independent trials and represented by symbols) and numerical evaluation of the theoretical expressions for the MSE in DOA (represented by linetypes), are shown to be close, validating the theory. The figures present the MSE in DOA $(-10 \log (M S E))$ in $\mathrm{dB}$ as a function of the number of subarrays $K$ for a two source scenario. Fig. 1 presents the MSE when there are only Gain errors in the array. and Fig. 2 for the case when there are only Phase errors in the array. It can be seen that the simulations match the theory closely. Figs. 3 and 4 compare the performance of FS and FBS for MUSIC for various values of correlation in the case of Gain errors. Similar trends are observed for all methods.

1. Theoretical expressions for the MSE in DOA as a function of the statistics of the array model errors for all subspace methods when spatial smoothing is used (FS and FBS) are obtained and verified using simulations. These are general expressions and are valid for various types of error models.

2. Two typical cases of error models, random errors and angle independent gain and phase errors have been studied in detail. For these error models, it is shown [8] that for the Forward case, $(K=1)$, the MSE for all methods is independent of the correlation between sources $(\rho)$ for $\rho<1$ (also seen in Figs. 3,4 ).
3. FBS improves the performance of the methods compared to FS (for $K>1$ ) for correlated sources, and is most significant for $\rho=1$ (also seen in Figs. $3,4)$.

4. In a one source example, all methods are insensitive to gain errors with smoothing. A similar result was shown for no smoothing case in [3].

5. For the cases considered, smoothing improves the performance of Min-Norm and ESPRIT. There exists an optimum value of $K$ for which the MSE is the lowest. Smoothing does not seem to improve the performance of MUSIC.

\section{REFERENCES}

[1] K. Wong, R. Walker, and G. Niezgoda, "Effects of random sensor motion on bearing estimation by the MUSIC algorithm," IEE Proc., Pt. F, vol. 135, pp. 233-250, June 1988.

[2] A. Swindlehurst and T. Kailath, "A Performance Analysis of Subspace-Based Methods in the presence of Model Errors - Part I: The MUSIC Algorithm," IEEE Trans. SP, vol. ASSP-40, pp. 17581774 , July 1992.

[3] H. Srinath and V. U. Reddy, "Analysis of MU. SIC algorithm with sensor gain and phase perturbations," SignalProcessing, vol. 23, pp. 245-256, 1991.

[4] F. Li and R. Vaccaro, "Statistical comparison of Subspace Based DOA estimation algorithms in the presence of sensor errors," in Proc. 5th ASSP Spectral Estimation Workshop, (Rochester, NY), pp. 327-331, Oct 1990.

[5] V. C. Soon and Y. F. Huang, "An Analysis of ESPRIT Under Random Sensor Uncertainties," IEEE Trans. SP, vol. ASSP-40, pp. 2353-2358, July 1992.

[6] Hari. V. S. Kuchibhotla, Performance Analysis of Subspace Based Methods for Direction Of Arrival ( DOA ) Estimation. PhD thesis, University of California, San Diego, La Jolla, CA, Sep 1990.

[7] B. D. Rao and K. V. S. Hari, "Effect of spatial smoothing on the performance of MUSIC and the minimum-norm method," IEE Proceedings - Part $F$, vol. 137, pp. 449-458, Dec. 1990.

[8] K. V. S. Hari and U. Gummadavelli, "Effect of Spatial smoothing on the Performance of Subspace methods in the Presence of Array Model Errors,". submitted to Automatica Special Issue on Statistical Signal Processing and Control. 

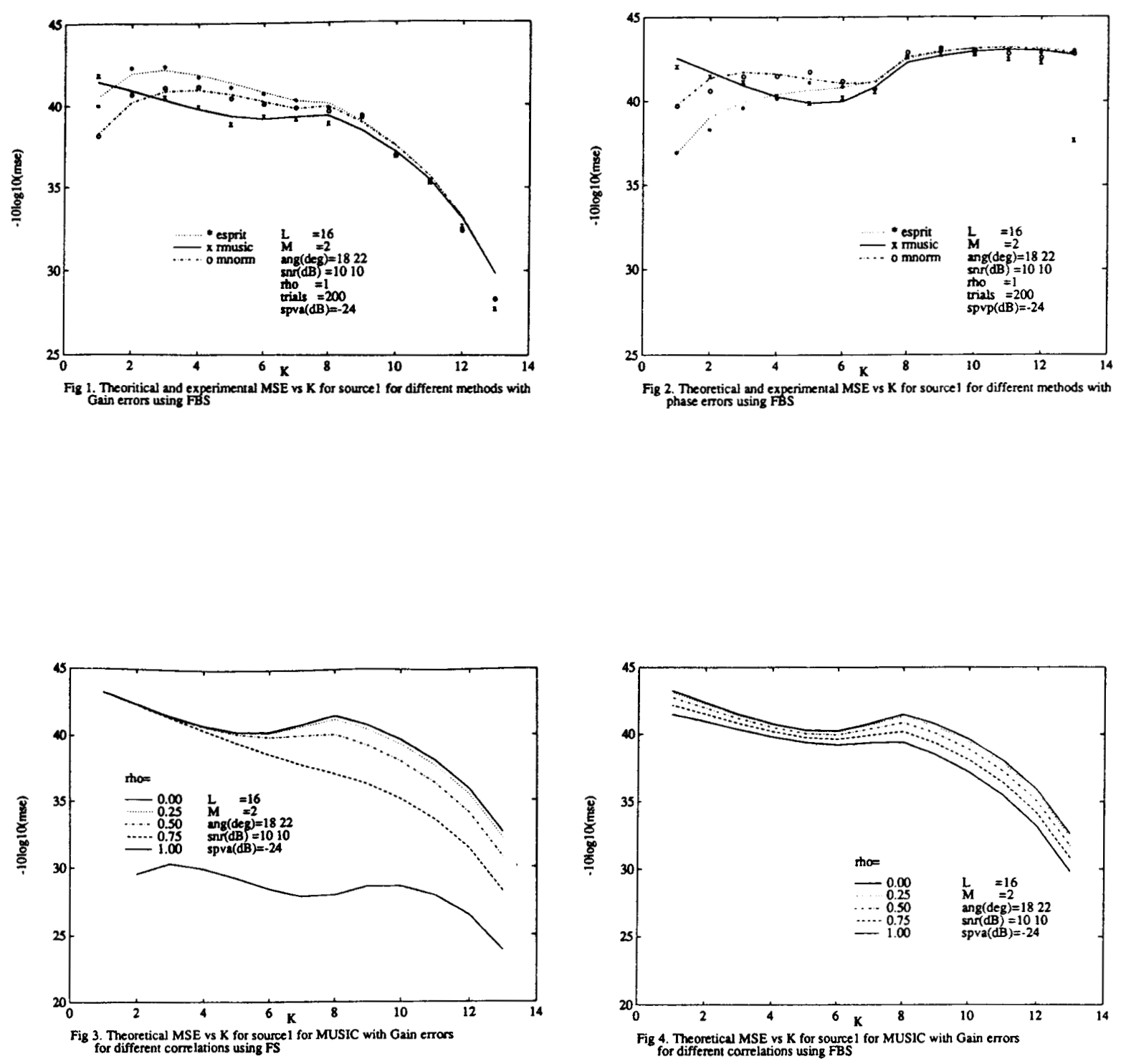

IV-355 